\title{
Optimal Tracking of Distributed Heavy Hitters and Quantiles
}

\author{
Ke Yi Qin Zhang \\ Department of Computer Science and Engineering \\ Hong Kong University of Science and Technology \\ \{yike, qinzhang\}cse.ust.hk
}

\begin{abstract}
We consider the the problem of tracking heavy hitters and quantiles in the distributed streaming model. The heavy hitters and quantiles are two important statistics for characterizing a data distribution. Let $A$ be a multiset of elements, drawn from the universe $U=\{1, \ldots, u\}$. For a given $0 \leq \phi \leq 1$, the $\phi$-heavy hitters are those elements of $A$ whose frequency in $A$ is at least $\phi|A|$; the $\phi$-quantile of $A$ is an element $x$ of $U$ such that at most $\phi|A|$ elements of $A$ are smaller than $A$ and at most $(1-\phi)|A|$ elements of $A$ are greater than $x$. Suppose the elements of $A$ are received at $k$ remote sites over time, and each of the sites has a two-way communication channel to a designated coordinator, whose goal is to track the set of $\phi$-heavy hitters and the $\phi$-quantile of $A$ approximately at all times with minimum communication. We give tracking algorithms with worst-case communication $\operatorname{cost} O(k / \epsilon \cdot \log n)$ for both problems, where $n$ is the total number of items in $A$, and $\epsilon$ is the approximation error. This substantially improves upon the previous known algorithms. We also give matching lower bounds on the communication costs for both problems, showing that our algorithms are optimal. We also consider a more general version of the problem where we simultaneously track the $\phi$-quantiles for all $0 \leq \phi \leq 1$.
\end{abstract}

\section{Introduction}

Data streams have been studied in both the database and theory communities for more than a decade $[2,3]$. In this model, data items arrive in an online fashion, and the goal is to maintain some function $f$ over all the items that have already arrived using small space. A lot of $f$ 's have been considered under the streaming model. The theory community have studied various frequency moments [2,20,31], geometric problems [1, 19,30], and some graph problems [5, 15]. While the database community have mostly focused on maintaining the frequent items (a.k.a. heavy hitters) $[8,10$, $22,25,26]$ and quantiles $[9,17,18]$, two very important statistics for characterizing a data distribution. Since we cannot afford to store all the items, we can only maintain an approximate $f$ (except for some trivial $f$ 's), and all the results in the streaming model are expressed as a tradeoff between the approximation error $\epsilon$ and the space used by the algorithm. After a long and somehow disorganized line of research, the heavy hitter problem is now completely understood with both space upper and lower bounds determined at $\Theta(1 / \epsilon)$; please see the recent paper by Cormode and Hadjieleftheriou [8] for a comprehensive comparison of the existing algorithms for this problem, both theoretically and empirically. For maintaining quantiles, the best upper bound is due to a sketch structure by Greenwald and Khanna [18], using space $O(1 / \epsilon \cdot \log (\epsilon n))$ where $n$ is the number of items in the stream. This is conjectured to be optimal but not yet proved.

Recent years have witnessed an increasing popularity of another model more general than the streaming model, where multiple streams are considered. In this model, multiple streams are received at multiple distributed sites, and again we would like to continuously track some function $f$ over the union of all the items that have arrived across all the sites. Here the most important measure of complexity is the total communication cost incurred during the entire tracking period. This model, which is either referred to as the distributed streaming model or the continuous communication model, is a natural combination of the classical communication model [32] and the data stream model. Recall that the communication model studies the problem of computing some function $f$ over distributed data using minimum communication. The data is predetermined and stored at a number of sites, which communicate with a 
central coordinator, and the goal is to do a one-time computation of the function $f$. Thus the distributed streaming model is more general as we need to maintain $f$ continuously over time as items arrive in a distributed fashion.

The rising interest on the distributed streaming model is mainly due to its many applications in distributed databases, wireless sensor networks, and network monitoring. As a result, it has attracted a lot of attention lately in the database community, resulting in a flurry of research in this area $[4,6,7,12-14,16,23,24,27-29]$. However, nearly all works in this area are heuristic and empirical in nature, with a few exceptions to be mentioned shortly. For many fundamental problems in this model, our theoretical understandings are still premature. This is to be contrasted with the standard streaming model, where theory and practice nicely blend, and in fact many of the most practically efficient solutions are the direct products of our theoretical findings. In this paper, we take an important step towards an analytical study of the distributed streaming model, by considering the worst-case communication complexity of tracking heavy hitters and quantiles, arguably two of the most fundamental problems on data streams.

The distributed streaming model. We now formally define the distributed streaming model, which is the same as in most works in this area. Let $A=\left(a_{1}, \ldots, a_{n}\right)$ be a sequence of items, where each item is drawn from the universe $U=\{1, \ldots, u\}$. The sequence $A$ is observed in order by $k \geq 2$ remote sites $S_{1}, \ldots, S_{k}$ collectively, i.e., item $a_{i}$ is observed by exactly one of the sites at time instance $t_{i}$, where $t_{1}<t_{2}<\cdots<t_{n}$. Let $A(t)$ be the multiset of items that have arrived up until time $t$ from all sites. Then the general goal is to continuously track $f(A(t))$ for some function $f$ at all times $t$ with minimum total communication among the sites. Note that in the classical communication model, the goal is to just compute $f(A(+\infty))$; in the data stream model, the goal is to track $f(A(t))$ for all $t$ but there is only one site $(k=1)$, and we are interested in the space complexity of the tracking algorithm, not communication. Thus, the distributed streaming model is a natural combination of the two, but is also significantly different from either.

We define the manner of communication more precisely as follows. There is a distinguished coordinator $C$, who will maintain (an approximate) $f(t)$ at all times. There is a two-way communication channel between the coordinator and each of the $k$ sites, but there is no direct communication between any two sites (but up to a factor of 2, this is not a restriction). Suppose site $S_{j}$ receives the item $a_{i}$ at time $t_{i}$. Based on its local status, $S_{j}$ may choose to send a message to $C$, which in turn may trigger iterative communication with other sites. We assume that communication is instant. When all communication finishes, all the sites who have been involved may have new statuses, getting ready for the next item $a_{i+1}$ to arrive. We will measure the communication cost in terms of words, and assume that each word consists of $\Theta(\log u)=\Theta(\log n)$ bits. Finally we assume that $n$ is sufficiently large (compared with $k$ and $1 / \epsilon)$; if $n$ is too small, a naive solution that transmits every arrival to the coordinator would be the best.

In this paper we will focus on the communication cost (or simply the cost). Nevertheless, all the algorithms proposed in this paper can be implemented both space- and time-efficiently.

Heavy hitters and quantiles. By taking different $f$ 's, we arrive at different continuous tracking problems. The notion of $\epsilon$-approximation also differs for different functions. We adopt the following agreed definitions in the literature. In the sequel, we abbreviate $A(t)$ as $A$ when there is no confusion.

For any $x \in U$, let $m_{x}(A)$ be the number of occurrences of $x$ in $A$. For some user specified $0 \leq \phi \leq 1$, the set of $\phi$-heavy hitters of $A$ is $\mathcal{H}_{\phi}(A)=\left\{x\left|m_{x}(A) \geq \phi\right| A \mid\right\}$, where $|A|$ denotes the total number of items in $A$. If an $\epsilon$-approximation is allowed, then the returned set of heavy hitters must contain $\mathcal{H}_{\phi}(A)$ and cannot include any $x$ such that $m_{x}(A)<(\phi-\epsilon)|A|$. If $(\phi-\epsilon)|A| \leq m_{x}(A)<\phi|A|$, then $x$ may or may not be reported. In the heavy hitter tracking problem, the coordinator should always maintain an approximate $\mathcal{H}_{\phi}(A)$ at all times for a given $\phi$.

For any $0 \leq \phi \leq 1$, the $\phi$-quantile of $A$ is some $x \in U$ such that at most $\phi|A|$ items of $A$ are smaller than $x$ and at most $(1-\phi)|A|$ items of $A$ are greater than $x$. The quantiles are also called order statistics in the statistics literature. In particular, the $\frac{1}{2}$-quantile is also known as the median of $A$. If an $\epsilon$-approximation is allowed, we can return any $\phi^{\prime}$-quantile of $A$ such that $\phi-\epsilon \leq \phi^{\prime} \leq \phi+\epsilon$. In the $\phi$-quantile tracking problem, the coordinator needs to keep an $\epsilon$-approximate $\phi$-quantile of $A$ at all times for a given $\phi$. We also consider a more general version of the problem, where we would like to keep track of all the quantiles approximately. More precisely, here the "function" $f$ is a data structure from which an $\epsilon$-approximate $\phi$-quantile for any $\phi$ can be extracted. Note that such a structure is equivalent to an (approximate) equal-height histogram, which characterizes the entire distribution.

In particular, from an all-quantile structure, we can easily obtain the $(2 \epsilon)$-approximate $\phi$-heavy hitters for any $\phi$, as observed in [7]. Therefore, the all-quantile tracking problem is more general than either the $\phi$-heavy hitter tracking problem or the $\phi$-quantile tracking problem. In the rest of the paper, we omit the word "approximate" when referring 
to heavy hitters and quantiles when the context is clear.

Previous works. Traditionally, query answering in distributed databases follows a "poll" based approach, that is, the coordinator collects information from the sites to answer a query posed by the user using minimum communication. Such a paradigm falls into the realm of the classical multi-party communication theory. These queries are also referred to as one-shot queries in the literature. As long-standing queries that need to be answered continuously become common in many modern applications such as sensor network monitoring, network anomaly detection, publish-subscribe systems, etc., periodically polling all the sites is neither efficient nor effective (i.e., long latency). Thus, the trend is moving towards a "push" based approach [21], in which the sites actively participate in the tracking process. In this framework, each site maintains some local conditions, and will not initiate communication unless one of the conditions is triggered. Such an approach often leads to much reduced communication overhead compared with the "poll" based approach, since the system will react only when "interesting" things are happening. This is the main motivation that has led to the distributed streaming model described above.

Various $f$ 's have been considered under this framework. The simplest case $f(A)=|A|$ just counts the total number of items received so far across all the sites. This problem can be easily solved with $O(k / \epsilon \cdot \log n)$ communication where each site simply reports to the coordinator whenever its local count increases by a $1+\epsilon$ factor [23]. The other important single-valued statistics are the frequency moments: $F_{p}(A)=\sum_{x}\left(m_{x}(A)\right)^{p}$. $F_{0}$ is the number of distinct items, and can be tracked with cost $O\left(k / \epsilon^{2} \cdot \log n \log \frac{n}{\delta}\right)$ [11]; $F_{2}$ is the self-join size and can be tracked with cost $O\left(\left(k^{2} / \epsilon^{2}+k^{3 / 2} / \epsilon^{4}\right) \log n \log \frac{k n}{\epsilon \delta}\right)$ [11]. Some heuristic approaches based on predicting future arrivals of items have been proposed in $[6,12]$.

Single-valued statistics have very limited expressive power, so multi-valued statistics are often necessary to better capture the distribution of data. The most important ones include the heavy hitters and quantiles, and they have also been studied under the distributed streaming framework. Babcock and Olston [4] designed some heuristics for the top- $k$ monitoring problem, where the goal is to track the $k$ most frequent items (whose frequency may not be larger than $\phi|A|$ ). Their techniques can be adapted to tracking the heavy hitters [16], but the approach remains heuristic in nature. Manjhi et al. [24] also studied the heavy hitter tracking problem, but their communication model and the goal are different: They organize the sites in a tree structure and the goal is to minimize the communication only at the root node. The all-quantile tracking problem has been studied by Cormode et al. [7], who gave an algorithm with cost $O\left(k / \epsilon^{2} \cdot \log n\right)$. As commented earlier, this also implies a heavy hitter tracking algorithm with the same cost. This remains the best communication upper bound for both problems to date. No lower bound is known.

Our results. Our main results in this paper are the matching upper and lower bounds on the communication cost for deterministic algorithms for both the heavy hitter tracking problem and the quantile tracking problem. Specifically, we show that for any $\phi$, both the $\phi$-heavy hitters (Section 2) and the $\phi$-quantile (Section 3) can be tracked with total communication $\operatorname{cost} O(k / \epsilon \cdot \log n)$. This improves upon the previous result of [7] by a $\Theta(1 / \epsilon)$ factor. We also give matching lower bounds for both problems, showing that our tracking protocols are optimal in terms of communication. Note that in the classical communication model, we can easily do a one-shot computation of the $\phi$-heavy hitters and the $\phi$-quantile easily with cost $O(k / \epsilon)$, as observed in [7]. Interestingly, our results show that requiring the heavy hitters and quantiles to be tracked at all times indeed increases the communication complexity, but only by a $\Theta(\log n)$ factor. In Section 4, we give an algorithm that tracks all quantiles with cost $O\left(k / \epsilon \cdot \log ^{2} \frac{1}{\epsilon} \log n\right)$. Because this problem is more difficult than the single-quantile problem, it has the same lower bound of $\Omega(k / \epsilon \cdot \log n)$ as the latter. Thus, our all-quantile tracking algorithm is also optimal up to a $\Theta\left(\operatorname{polylog} \frac{1}{\epsilon}\right)$ factor.

\section{Tracking the Heavy Hitters}

\subsection{The upper bound}

The algorithm. Let $m$ be the current size of $A$. First, the coordinator $C$ always maintains $C$. $m$, an $\epsilon$-approximation of $m$. This can be achieved by letting each site send its local count every time it has increased by a certain amount (to be specified shortly). Each site $S_{j}$ maintains the exact frequency of each $x \in U$ at site $S_{j}$, denoted $m_{x, j}$, at all times. The overall frequency of $x$ is $m_{x}=\sum_{j} m_{x, j}$. Of course, we cannot afford to keep track of $m_{x}$ exactly. Instead, the coordinator $C$ maintains an underestimate $C . m_{x, j}$ of $m_{x, j}$, and sets $C . m_{x}=\sum_{j} C . m_{x, j}$ as an estimate of $m_{x}$. 
$S_{j}$ will send its local increment of $m_{x, j}$ to $C$, hence updating $C . m_{x, j}$, from time to time following certain rules to be specified shortly. In addition, each site $S_{j}$ maintains $S_{j} . m$, an estimate of $m$, a counter $S_{j} . \Delta(m)$, denoting the increment of $S_{j} . m$ since its last communication to $C$ about $S_{j} . m$, as well as a counter $S_{j} . \Delta\left(m_{x}\right)$ for each $x$, denoting the increment of $S_{j} . m_{x}$ since its last communication to $C$ about $m_{x, j}$.

We can assume that the system starts with $m=k / \epsilon$ items; before that we could simply send each item to the coordinator. So when the algorithm initiates, all the estimates are exact. We initialize $S_{j} . \Delta(m)$ and $S_{j} . \Delta\left(m_{x}\right)$ for all $x$ to be 0 . The protocols of tracking the $\phi$-heavy hitters are as follows.

1. Each site $S_{j}$ : When a new item of $x$ arrives, $S_{j} . \Delta(m)$ and $S_{j} . \Delta\left(m_{x}\right)$ are incremented by 1 . When $S_{j} . \Delta(m)$ (resp. $\left.S_{j} . \Delta\left(m_{x}\right)\right)$ reaches $\left(\epsilon \cdot S_{j} \cdot m\right) / 3 k$, site $S_{j}$ sends a message $\left(a l l,\left(\epsilon \cdot S_{j} \cdot m\right) / 3 k\right)\left(\right.$ resp. $\left(x,\left(\epsilon \cdot S_{j} \cdot m\right) / 3 k\right)$ ) to the coordinator, and resets $S_{j} . \Delta(m)$ (resp. $S_{j} . \Delta\left(m_{x}\right)$ ) to 0 .

2. Coordinator: When $C$ has received a message $\left(a l l,\left(\epsilon \cdot S_{j} . m\right) / 3 k\right)$ or $\left(x,\left(\epsilon \cdot S_{j} \cdot m\right) / 3 k\right)$, it updates $C . m$ to $C . m+\left(\epsilon \cdot S_{j} . m\right) / 3 k$ or $C . m_{x}$ to $C . m_{x}+\left(\epsilon \cdot S_{j} . m\right) / 3 k$, respectively. Once $C$ has received $k$ signals in the forms of $\left(a l l,\left(\epsilon \cdot S_{j} . m\right) / 3 k\right)$, it collects the local counts from each site to compute the exact value of $m$, sets $C . m=m$, and then broadcasts $C . m$ to all sites. Then each site $S_{j}$ updates its $S_{j} . m$ to $m$. After getting a new $S_{j} . m, S_{j}$ also resets $S_{j} . \Delta(m)$ to 0 .

Finally, at any time, the coordinator $C$ declares an item $x$ to be a $\phi$-heavy hitter if and only if

$$
\frac{C . m_{x}}{C . m} \geq \phi+\frac{\epsilon}{2}
$$

Correctness. To prove correctness we first establish the following invariants maintained by the algorithm.

$$
\begin{gathered}
m_{x}-\frac{\epsilon m}{3}+k \leq C . m_{x} \leq m_{x}, \\
m-\frac{\epsilon m}{3}+k \leq C . m \leq m .
\end{gathered}
$$

The second inequalities of both (3) and (2) are obvious. The first inequality of (2) is valid since once a site $S_{j}$ gets $\left(\epsilon \cdot S_{j} . m\right) / 3 k$ items of $x$, it sends a message to the coordinator and the coordinator updates $C . m_{x}$ accordingly. Thus the maximum error of $C . m$ in the coordinator is at most $\sum_{j=1}^{k}\left(\frac{\epsilon \cdot S_{j} . m}{3 k}-1\right) \leq \frac{\epsilon m}{3}-k$. The first inequality of (3) follows from a similar reason. Combining (2) and (3), we have

$$
\frac{m_{x}}{m}-\frac{\epsilon}{3}<\frac{C . m_{x}}{C . m}<\frac{m_{x}}{m} \cdot \frac{1}{1-\epsilon / 3}<\frac{m_{x}}{m}+\frac{\epsilon}{2},
$$

which guarantees that the approximate ratio $\frac{C . m_{x}}{C . m}$ is within $\epsilon / 2$ of $\frac{m_{x}}{m}$, thus classifying an item using (1) will not generate any false positives or false negatives.

Analysis of communication complexity. We divide the whole tracking period into rounds. A round start from the time when the coordinator finishes a broadcast of $C . m$ to the time when it initiates the next broadcast. Since the coordinator initiates a broadcast after $C . m$ is increased by a factor of $1+\sum_{i=1}^{k}(\epsilon / 3 k)=1+\epsilon / 3$, the number of rounds is bounded by

$$
\log _{1+\epsilon / 3} n=O\left(\frac{\log n}{\epsilon}\right) .
$$

In each round, the number of messages in the form of $\left(a l l,\left(\epsilon \cdot S_{j} . m\right) / 3 k\right)$ sent by all the sites is $k$ by the definition of our protocol. Since there are $O(\log n / \epsilon)$ rounds in total, the number of messages in the form of $\left(a l l,\left(\epsilon \cdot S_{j} . m\right) / 3 k\right)$ can be bounded by $O(k / \epsilon \cdot \log n)$. On the other hand, it is easy to see that total number of messages of the form $\left(x,\left(\epsilon \cdot S_{j} \cdot m\right) / 3 k\right)$ is no more than the total number of messages of the form $\left(a l l,\left(\epsilon \cdot S_{j} \cdot m\right) / 3 k\right)$. Therefore, the total cost of the whole system is bounded by $O(k / \epsilon \cdot \log n)$.

Theorem 2.1 For any $\epsilon \leq \phi \leq 1$, there is a deterministic algorithm that continuously tracks the $\phi$-heavy hitters and incurs a total communication cost of $O(k / \epsilon \cdot \log n)$. 
Implementing with small space. In the algorithm described above, we have assumed that each site maintains all of its local frequencies $S_{j} \cdot m_{x}$ exactly. In fact, it is not difficult to see that our algorithm still works if we replace these exact frequencies with a heavy hitter sketch, such as the space-saving sketch [26], that maintains the local $\epsilon^{\prime}$ approximate frequencies for all items for some $\epsilon^{\prime}=\Theta(\epsilon)$. More precisely, such a sketch gives us an approximate $S_{j} . m_{x}$ for any $x \in U$ with absolute error at most $\epsilon^{\prime}\left|S_{j}\right|$, where $\left|S_{j}\right|$ denotes the current number of items received at $S_{j}$ so far. We need to adjust some of the constants above, but this does not affect our asymptotic results. By using such a sketch at each site, our tracking algorithm can be implemented in $O(1 / \epsilon)$ space per site and amortized $O(1)$ time per item.

\subsection{The lower bound}

To give a lower bound on the total communication cost that any deterministic tracking algorithm must take, we first consider the number of changes that the set of heavy hitters could experience, where a change is defined to be the transition of the frequency of an item from above $\phi|A|$ to below $(\phi-\epsilon)|A|$, or the other way round. Then we show that to correctly detect each change, the system must exchange at least a certain amount of messages. The following lemma could be established by construction.

Lemma 2.2 For any $\phi>3 \epsilon$, there is a sequence of item arrivals such that the set of heavy hitters in the whole tracking period will have $\Omega(\log n / \epsilon)$ changes.

Proof: Set $\epsilon^{\prime}=2 \epsilon$. We construct two groups of $l=1 /\left(2 \phi-\epsilon^{\prime}\right)$ items each: $\mathcal{S}_{0}=\left\{t_{1}, t_{2}, \ldots, t_{l}\right\}$ and $\mathcal{S}_{1}=$ $\left\{t_{l+1}, t_{l+2}, \ldots, t_{2 l}\right\}$. Since we only care about the total number of changes of the set of heavy hitters during the whole tracking period, we temporarily treat the whole system as one big site and items come one by one. We will construct an input sequence under which the set of heavy hitters will undergo $\Omega(\log n / \epsilon)$ changes.

We still divide the whole tracking period to several rounds, and let $m_{i}$ denote the total number of items when round $i$ starts. The following invariant will be maintained throughout the construction:

Let $b=i \bmod 2$. When round $i$ starts, all items $t \in \mathcal{S}_{b}$ have frequency $\phi m_{i}$, and all items $t \in \mathcal{S}_{1-b}$ have frequency $\left(\phi-\epsilon^{\prime}\right) m_{i}$.

It can be verified that the total frequency of all items is indeed $m_{i}$. Note that from the start of round $i$ to the end of round $i$, all the non-heavy hitters become heavy hitters, and all the heavy hitters become non-heavy hitters. In what follows we only care about the changes of the former type, which lower bounds the number of changes. To maintain the invariant for round $i+1$, we construct item arrivals as follows. Without loss of generality, suppose $\mathcal{S}_{1-b}=\left\{t_{1}, t_{2}, \ldots, t_{l}\right\}$. Let $\beta=\frac{\epsilon^{\prime}\left(2 \phi-\epsilon^{\prime}\right)}{\phi-\epsilon^{\prime}}$. We first generate $\beta m_{i}$ copies of $t_{1}$, and then $\beta m_{i}$ copies of $t_{2}, \ldots$, then $\beta m_{i}$ copies of $t_{l}$, in sequence. After these items we end round $i$ and start round $i+1$. At this turning point, the total number of items is

$$
m_{i+1}=m_{i}+l \cdot \beta m_{i}=\frac{\phi}{\phi-\epsilon^{\prime}} m_{i}
$$

Now the frequency of each item in the set $\mathcal{S}_{1-b}$ is

$$
\left(\phi-\epsilon^{\prime}\right) m_{i}+\beta m_{i}=\phi \cdot \frac{\phi}{\phi-\epsilon^{\prime}} m_{i}=\phi m_{i+1},
$$

and the frequency of each item in $\mathcal{S}_{b}$ remains the same, that is, $\phi m_{i}=\left(\phi-\epsilon^{\prime}\right) m_{i+1}$. Now we have restored the invariant and can start round $i+1$.

Finally, we bound the number of rounds. Since the total number of items $m_{i}$ increases by a $\phi /\left(\phi-\epsilon^{\prime}\right)$ factor in each round, the total number of rounds is $\Theta\left(\log _{\frac{\phi}{\phi-\epsilon^{\prime}}} n\right)$. Consequently, the total number of changes in the set of heavy hitters (from non-heavy hitters to heavy hitters) is $l \cdot \Theta\left(\log _{\frac{\phi}{\phi-\epsilon^{\prime}}} n\right)=\Omega\left(\frac{1}{\phi-\epsilon} \cdot \frac{\phi-\epsilon^{\prime}}{\epsilon^{\prime}} \log n\right)=\Omega(\log n / \epsilon)$.

Now we go back to the distributed scenario and consider the cost of communication for "recognizing" each change. Because we allow some approximation when classifying heavy hitters and non-heavy hitters, the valid time to report a change is actually a time interval, from the time when its frequency just passes $(\phi-\epsilon)|A|$ to the time when its 
frequency reaches $\phi|A|$. As long as the tracking algorithm signals the change within this interval, the algorithm is considered to be correct. Consider the construction in the proof of Lemma 2.2. In round $i$, the transition interval from a non-heavy hitter to a heavy hitter for an item $t$ must lie inside the period in which the $\beta m_{i}$ copies of $t$ arrive. Below we will show that in order for the coordinator to signal the change within this period, $\Omega(k)$ messages have to be exchanged in the worst case using an adversary argument.

Before presenting the lower bound proof, let us be more precise about the computation model. Recall that in the introduction, the model forbids a site to spontaneously initiate communication or change its local status; actions can only be triggered as a result of the arrival of an item at this site, or in response to the coordinator. Note that for deterministic algorithms this is not a restrictive assumption. In our case, since we only care about the frequency of a particular item $t$ increasing from $m_{i}$ to $m_{i}+\beta m_{i}$, we may assume that each site $S_{j}$ has a triggering threshold $n_{j}$, meaning that $S_{j}$ will only initiate communication when the number of copies of $t$ received by $S_{j}$ is $n_{j}$. When all the communication triggered by the arrival of an item finishes, all the sites that have participated are allowed to update their triggering thresholds, but the rest of the sites must retain their old thresholds.

Lemma 2.3 To correctly recognize a change in the heavy hitters under the input constructed in the proof of lemma 2.2, any deterministic algorithm has to incur a communication cost of $\Omega(k)$.

Proof: We will construct an adversary who will send the $\beta m_{i}$ copies of $t$ to the sites in a way such that at least $\Omega(k)$ sites must communicate with the coordinator. Since we are dealing with deterministic algorithms, we may assume that the adversary knows the triggering thresholds $n_{j}$ at any time.

Initially, we must have

$$
\sum_{j=1}^{k}\left(n_{j}-1\right)<\beta m_{i} .
$$

Otherwise, the adversary can send $n_{j}-1$ copies to $S_{j}$ for all $j$ without triggering any communication, and make the algorithm miss the change. Therefore there must be some $j$ such that $n_{j} \leq \beta m_{i} / k+1 \leq 2 \beta m_{i} / k$. The adversary first sends $2 \beta m_{i} / k$ copies of $t$ to $S_{j} . S_{j}$ will then communicate with the coordinator at least once. After the first $2 \beta m_{i} / k$ copies, the new triggering thresholds must still satisfy (4). Similarly, there is some $n_{j^{\prime}} \leq 2 \beta m_{i} / k$, and the adversary will send another $2 \beta m_{i} / k$ copies of $t$ to $S_{j^{\prime}}$. Such a process can be repeated for $\frac{\beta m_{i}}{2 \beta m_{i} / k}=\Omega(k)$ times, triggering at least $\Omega(k)$ messages of communication.

The following lower bound follows immediately from Lemma 2.2 and Lemma 2.3, for the reason that the tracking algorithm has to correctly and continuously maintain the whole set of heavy hitters.

Theorem 2.4 Any deterministic algorithm that continuously tracks the $\phi$-heavy hitters has to incur a total communication cost of $\Omega(k / \epsilon \cdot \log n)$, for any $\phi>3 \epsilon$.

Remark. Note that our lower bound above is actually lower bound on the number of messages required. Also recall that our algorithm in Section 2.1 sends $O(k / \epsilon \cdot \log n)$ messages and each message if of constant size. Our lower bound implies that one cannot hope to reduce the number of messages by making each of them longer.

\section{Tracking the Median}

In this section we first present an algorithm to track any $\phi$-quantile for $0 \leq \phi \leq 1$. For ease of presentation we describe how to track the median (the 1/2-quantile); the generalization to any $\phi$-quantile is straightforward. Then we give a matching lower bound.

\subsection{The upper bound}

For simplicity we assume that all the items in $A$ are distinct; issues with ties can be easily resolved by standard techniques such as symbolic perturbation. We divide the whole tracking period into $O(\log n)$ rounds; whenever $|A|$ 
doubles, we start a new round. In the following we focus on one round, and show that our median-tracking algorithm has a communication cost of $O(k / \epsilon)$.

Let $m$ be the cardinality of $A$ at the beginning of a round. Note that $m$ is fixed throughout a round and we always have $m \leq|A|$. The main idea of our algorithm is to maintain a dynamic set of disjoint intervals in the coordinator (by maintaining a set of separating items), such that each interval contains between $\frac{\epsilon}{8} m$ and $\frac{\epsilon}{2} m$ items. We first show that if we have such a set of intervals, the median can be tracked efficiently. Afterward we discuss how to maintain these intervals.

Let $M$ denote the approximate median that is kept at the coordinator. We maintain two counters $C . \Delta(L)$ and $C . \Delta(R)$, counting the number of items that have been received at all sites to the left and the right of $M$, respectively. These two counters are maintained as underestimates with an absolute error at most $\frac{\epsilon}{8} m$, by asking each site to send in an update whenever it has received $\frac{\epsilon}{8 k} m$ items to the left or right of $M$. So the cost of maintaining them is $O(k / \epsilon)$.

Whenever $|C . \Delta(L)-C . \Delta(R)| \geq \frac{\epsilon}{2} m$, we update $M$ as follows.

1. Compute $C . L$ and $C . R$ as the total number of items to the left and the right of M. W.1.o.g., suppose $C . L>C . R$ and let $d=(C . L-C . R) / 2$.

2. Compute a new median $M^{\prime}$ such that $\left|r(M)-r\left(M^{\prime}\right)-d\right| \leq \frac{\epsilon}{4} m$ where $r(M)$ is the rank of $M$ in $A$. Update $M$ to $M^{\prime}$. Note that $M^{\prime}$ is at most $\frac{\epsilon}{4} m$ items away from the exact median. We will describe how to compute such an $M^{\prime}$ shortly.

3. Reset $C . \Delta(L)$ and $C . \Delta(R)$ to 0 .

For the correctness of the algorithm, we can show that our tracking algorithm always maintains an approximate median that is at most $\frac{\epsilon}{4} m+\frac{3 \epsilon}{4} m=\epsilon m$ items away from the exact median. The first term $\frac{\epsilon}{4} m$ is due to the fact that whenever we update $M, M$ is within an error of at most $\frac{\epsilon}{4} m$ to the exact median. The second term $\frac{3 \epsilon}{4} m$ accounts for the error introduced by the triggering condition $|C . \Delta(L)-C . \Delta(R)|$ monitored in the coordinator. Note that we keep both $C . \Delta(L)$ and $C . \Delta(R)$ within an additive error of at most $\frac{\epsilon}{8} m$ and whenever $|C . \Delta(L)-C . \Delta(R)| \geq \frac{\epsilon}{2} m$, we initiate an update. Therefore, the total error introduced is at most $2 \cdot \frac{\epsilon}{8} m+\frac{\epsilon}{2} m=\frac{3 \epsilon}{4} m$.

Now we analyze the communication cost. Step 1 could be done by exchanging $O(k)$ messages. For step 2, first note that $d \leq \epsilon m$ since by the reasoning above, $M$ is still an $\epsilon$-approximate median. Next, we can find $M^{\prime}$ quickly with the help of the set of intervals. We start by finding the first separating item $Y_{1}$ of the intervals to the left of $M$, and then collect information from all sites to compute the number of items in the interval $\left[Y_{1}, M\right]$, say $n_{1}$. If $\left|n_{1}-d\right| \leq \frac{\epsilon}{2} m$, we are done; otherwise we go on to pick the second separating item $Y_{2}$ to the left of $M$, and check if $\left|n_{2}-d\right| \leq \frac{\epsilon}{2} m$, where $n_{2}$ is the number of items in the interval $\left[Y_{2}, M\right]$. It is easy to see that after at most $O(1)$ such probes, we can find an item $Y$ such that the rank difference between $Y$ and the exact median is no more than $\frac{\epsilon}{2} m$. Note that the cost of each probe is $O(k)$ thus the total cost of step 2 is $O(k)$. Finally, we update $M$ at most $O(1 / \epsilon)$ times within a single round, since each update increases $|A|$ by at least a factor of $1+\frac{\epsilon}{2}$. To sum up, the total cost of the algorithm within a round is $O(k / \epsilon)$ provided that the dynamic set of intervals are maintained.

Maintaining the set of intervals. When a new round starts, we initialize the set of intervals as follows: Each site $S_{j}(1 \leq j \leq k)$ computes a set of intervals, each containing $\frac{\epsilon\left|A_{j}\right|}{32}$ items, where $A_{j}$ stands for the set of items $S_{j}$ has received, and then sends the set of intervals to the coordinator (by sending those separating items). Then the coordinator can compute the rank of any $x \in U$ with an error of at most $\sum_{j=1}^{k} \frac{\epsilon}{32}\left|A_{j}\right|=\frac{\epsilon}{32} m$, therefore it can compute a set of intervals, each of which contains at least $\frac{\epsilon}{8} m$ and at most $\frac{\epsilon}{4} m$ items. After the coordinator has built the set of intervals, it broadcasts them to all the $k$ sites, and then computes the exact number of items in each interval. The cost of each rebuilding is $O(k / \epsilon)$.

During each round, each site $S_{j}$ maintains a counter for each interval as new items arrive. And whenever the local counter of items in some interval $I$ has increased by $\frac{\epsilon}{4 k} m$, it sends a message to the coordinator and the coordinator updates the count for interval $I$ accordingly. Whenever the count of some interval in the coordinator $C$ reaches $\frac{\epsilon}{4} m$, the coordinator splits the interval into two intervals, each of which containing at least $\frac{\epsilon}{8} m$ and at most $\frac{\epsilon}{4} m$ items. To perform such a split, we can again call the rebuilding algorithm above, except that the rebuilding is only applied to the interval $I$, so the cost is only $O(k)$.

The correctness of algorithm is obvious. The total communication cost of interval splits is $O(k / \epsilon)$ in each round, since there are at most $O(1 / \epsilon)$ splits and each split incurs a communication cost $O(k)$. 
Theorem 3.1 There is a deterministic algorithm that continuously tracks the $\epsilon$-approximate median (and generally, any $\phi$-quantile $(0 \leq \phi \leq 1)$ ) and incurs a total communication cost of $O(k / \epsilon \cdot \log n)$.

Implementing with small space. Similar to our heavy hitter tracking algorithm, instead of maintaining the intervals exactly at each site, we can again deploy a sketch that maintains the approximate $\epsilon^{\prime}$-quantiles for some $\epsilon^{\prime}=\Theta(\epsilon)$ to maintain these intervals approximately. Suppose we use the Greenwald-Khanna sketch [18], then we can implement our $\phi$-quantile tracking algorithm with $O(1 / \epsilon \cdot \log (\epsilon n))$ space per site and amortized $O(\log n)$ time per item.

\subsection{The lower bound}

The idea of the proof of the lower bound is similar as that for the heavy hitters. We try to construct a sequence of input with the following properties.

1. The median will change at least $\Omega(\log n / \epsilon)$ times.

2. To correctly recognize each update, any deterministic algorithm has to incur a communication cost of $\Omega(k)$.

Consider the following construction. The universe consists of only two items 0 and 1 . We divide the whole tracking period to several rounds and let $m_{i}$ be the number of items at the beginning of round $i$. We maintain the following invariant: When round $i$ starts, the frequency of item $b$ is $(0.5-2 \epsilon) m_{i}$ and the frequency of item $1-b$ is $(0.5+2 \epsilon) m_{i}$, where $b=i \bmod 2$. This could be done by inserting $\frac{4 \epsilon}{0.5-2 \epsilon} m_{i}$ copies of $b$ during round $i$ and then start a new round. It is easy to see that there will be at least $\Omega(\log n / \epsilon)$ rounds and the median will change at least once during each round, therefore the total number of changes of the median is $\Omega(\log n / \epsilon)$. For the second property, we can invoke the same arguments as that for Lemma 2.3. Combining the two properties, we have the following.

Theorem 3.2 Any deterministic algorithm that continuously tracks the approximate median has to incur a total communication cost of $\Omega(k / \epsilon \cdot \log n)$.

\section{Tracking All Quantiles}

In this section, we give a tracking algorithm so that the coordinator $C$ always tracks the $\epsilon$-approximate $\phi$-quantiles for all $0 \leq \phi \leq 1$ simultaneously. We will solve the following equivalent problem: The coordinator is required to maintain a data structure from which we can extract the rank $r(x)$ for any $x \in U$ in $A$ with an additive error at most $\epsilon|A|$. We still assume that all items in $A$ are distinct.

We divide the whole tracking period into $O(\log n)$ rounds. In each round $|A|$ roughly doubles. We will show that the algorithm's cost in each round is $O\left(k / \epsilon \cdot \log ^{2} \frac{1}{\epsilon}\right)$. The algorithm restarts itself at the beginning of each round, therefore the total communication of the algorithm will be $O\left(k / \epsilon \cdot \log n \log ^{2} \frac{1}{\epsilon}\right)$.

The data structure. Let $m$ be the cardinality of $A$ at the beginning of a round. The data structure is a binary tree $\mathcal{T}$ with $\Theta(1 / \epsilon)$ leaves. The root $r$ of $\mathcal{T}$ corresponds to the entire $A$. It stores a splitting element $x_{r}$ which is an approximate median of $A$, i.e., it divides $A$ into two parts, either of which contains at least $\left(\frac{1}{2}-\alpha\right)|A|$ and at most $\left(\frac{1}{2}+\alpha\right)|A|$ items, for some constant $0<\alpha<\frac{1}{2}$. Then we recursively build $r$ 's left and right subtrees on these two parts respectively, until there are no more than $\epsilon m / 2$ items left. It is clear that $\mathcal{T}$ has $\Theta(1 / \epsilon)$ nodes in total, and has height at most $h=\log _{\frac{1}{2}+\alpha} \frac{\epsilon}{2}=\Theta\left(\log \frac{1}{\epsilon}\right)$, though it is not necessarily balanced. Each node in $\mathcal{T}$ is naturally associated with an interval. Let $I_{u}$ be the interval associated with $u$. Then $I_{r}$ is the entire $U$; suppose $v$ and $w$ are $u$ 's children, then $I_{u}$ is divided into $I_{v}$ and $I_{w}$ by $x_{u}$. Set $\theta=\frac{\epsilon}{2 h}$. Each node $u$ of $\mathcal{T}$ is in addition associated with $s_{u}$, which is an underestimate of $\left|A \cap I_{u}\right|$ with an absolute error of at most $\theta m$, i.e., $\left|A \cap I_{u}\right|-\theta m \leq s_{u} \leq\left|A \cap I_{u}\right|$. Please see Figure 1 for an illustration of the data structure.

If the coordinator has such a data structure, it is not difficult to see that we can compute the rank of $x$ with an absolute error of at most $\epsilon m$. For a given $x$, we first search down the binary tree and locate the leaf $v$ such that $x \in I_{v}$. As we go along the root-to-leaf path, whenever we follow a right child, we add up the $s_{u}$ of its left sibling. In the end we add up $h$ such partial sums, each contributing an error of at most $\theta m$, totaling $\theta m \cdot h=\epsilon m / 2$. Finally, since $\left|A \cap I_{v}\right|<\epsilon m / 2$, the sum of all the $s_{u}$ 's for the preceding intervals of $x$ is off by at most $\epsilon m$ from the actual rank of $x$. 


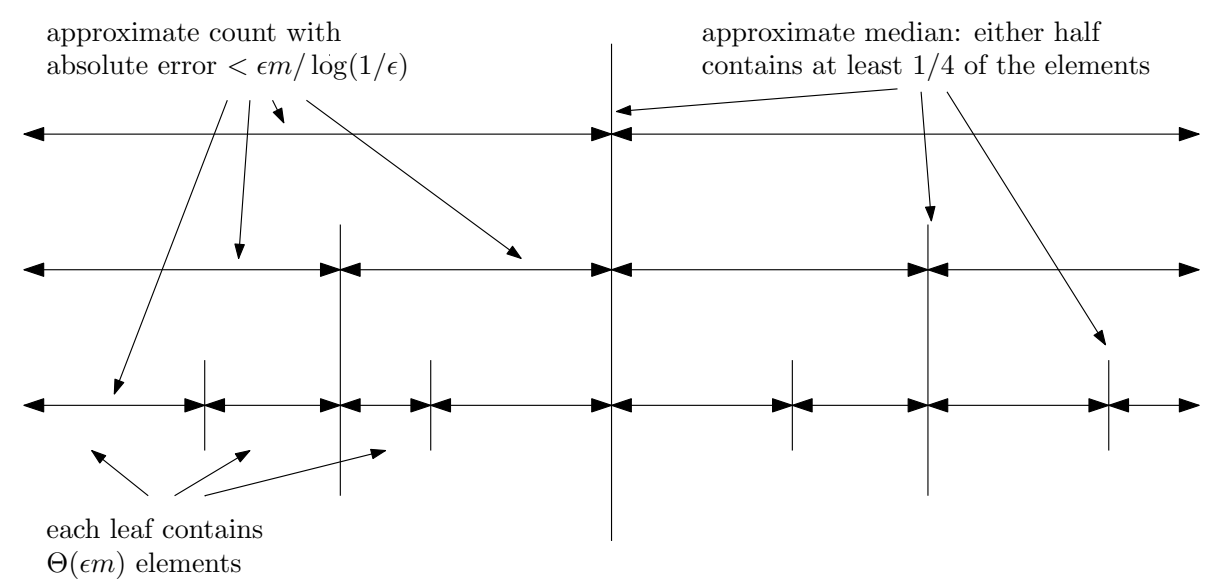

Figure 1: The data structure that can be used to extract the rank of any $x$ with absolute error $<\epsilon m$.

Initialization. At the beginning of each round, we initialize the data structure similarly as in Section 3. Suppose the set of items at $S_{j}$ is $A_{j}$. Each site $S_{j}$ builds its own structure $S_{j} . \mathcal{T}$, but with $\epsilon / 32$ as the error parameter, and ships to $C$. This costs a communication of $O(k / \epsilon)$. Note that $S_{j} \cdot \mathcal{T}$ allows one to extract the rank of any $x$ within $A_{j}$ with an error of $\epsilon / 32 \cdot\left|A_{j}\right|$. By querying each $S_{j} . \mathcal{T}$, the coordinator can compute the rank of any $x$ with an error of $\sum_{i=1}^{k} \frac{\epsilon}{32}\left|A_{i}\right|=\frac{\epsilon}{32} m$, which is enough for the coordinator to build its own $C . \mathcal{T}$. In particular, all the splitting elements can be chosen to be within a distance of $\frac{\epsilon}{32} m$ to the real median. After building $C \cdot \mathcal{T}$, the coordinator broadcasts it to all the sites, costing communication $O(k / \epsilon)$. Now each site $S_{j}$ knows how $U$ is subdivided into those $\Theta(1 / \epsilon)$ intervals represented by the binary tree $\mathcal{T}$. Then for each interval $I_{u}$, it computes $\left|A_{j} \cap I_{u}\right|$ and sends the count to $C$, so that the coordinator has all the exact partial sums $s_{u}$ to start with. It is easy to see that the total communication cost for initializing the data structure is $O(k / \epsilon)$.

Maintaining the partial sums. As items arrive, each site $S_{j}$ monitors all the intervals $I_{u}$ in $\mathcal{T}$. For each $I_{u}$, every time the local count of items in $I_{u}$ at $S_{j}$ has increased by $\theta m / k$, it sends an updated local count to $C$. Thus in the worst case, each site is holding $(\theta m / k-1)$ items that have not been reported, leading to a total error of at most $\theta m$. The cost of these messages can be bounded as follows. When $S_{j}$ sends a new count for some interval $I_{u}$, we charge the cost to the $\theta m / k$ new items that have arrived since the last message for $I_{u}, O(k /(\theta m))$ each. Since each item contributes to the counts of at most $h$ intervals, it is charged $O(h)$ times, so the total cost charged to one item is $O\left(\frac{k h}{\theta m}\right)$. There are a total of $O(m)$ items in a single round, so the overall cost is $O(k h / \theta)=O\left(k / \epsilon \cdot \log ^{2} \frac{1}{\epsilon}\right)$.

Maintaining the splitting elements. The maintenance algorithm above ensures that all the $s_{u}$ are within the desired error bound. We still need to take care of all the splitting elements, making sure that they do not deviate from the real medians too much. Specifically, when we build $\mathcal{T}$, for any $u$ with children $v$ and $w$, we ensure that

$$
\frac{3}{8}\left|A \cap I_{u}\right| \leq\left|A \cap I_{v}\right| \leq \frac{5}{8}\left|A \cap I_{u}\right| .
$$

This property can be easily established during initialization, since $\left|A \cap I_{u}\right|>\frac{\epsilon}{2} m$ for any internal node $u$ of $\mathcal{T}$, and we can estimate $\left|A \cap I_{v}\right|$ with an error of $\frac{\epsilon}{32} m$. In the middle of the round, we maintain the following condition:

$$
\frac{1}{4} s_{u} \leq s_{v} \leq \frac{3}{4} s_{u}
$$

Recall that $s_{u}$ (resp. $s_{v}$ ) is an estimate of $\left|A \cap I_{u}\right|$ (resp. $\left|A \cap I_{v}\right|$ ) with an error of at most $\theta m$. As long as (6) holds, we have

$$
\frac{1}{4}\left(\left|A \cap I_{u}\right|-\theta m\right) \leq \frac{1}{4} s_{u} \leq s_{v} \leq\left|A \cap I_{v}\right|+\theta m .
$$


Rearranging,

$$
\left|A \cap I_{v}\right| \geq \frac{1}{4}\left|A \cap I_{u}\right|-\frac{5}{4} \cdot \frac{\epsilon}{2 h} m \geq \frac{1}{4}\left|A \cap I_{u}\right|-\frac{5}{4} \cdot \frac{1}{h}\left|A \cap I_{u}\right| \geq \frac{3}{32}\left|A \cap I_{u}\right|,
$$

for $h \geq 8$. (Note that assuming $h$ larger than any constant does not affect our asymptotic results.) Similarly, we also have $\left|A \cap I_{v}\right| \leq \frac{29}{32}\left|A \cap I_{u}\right|$. Thus condition (6) ensures that the height of $\mathcal{T}$ is bounded by $h=\Theta\left(\log \frac{1}{\epsilon}\right)$.

Whenever (6) is violated, we do a partial rebuilding of the subtree rooted at $u$ to restore this condition. If multiple conditions are violated at the same time, we rebuild at the highest such node. To rebuild the subtree rooted at $u$, we apply our initialization algorithm, but only for the range $I_{u}$. This incurs a cost of $O\left(k \frac{\left|A \cap I_{u}\right|}{\epsilon m}\right)$, since we are essentially building a new data structure on $\left|A \cap I_{u}\right|$ elements with error parameter $\epsilon^{\prime}=\epsilon m /\left|A \cap I_{u}\right|$. After rebuilding, we have restored (5) for $u$ and all its descendants.

It remains to bound the cost of the partial rebuildings. Similarly as before, we can show that when (6) is violated, we must have

$$
\left|A \cap I_{v}\right|<\frac{21}{64}\left|A \cap I_{u}\right|
$$

or

$$
\left|A \cap I_{v}\right|>\frac{43}{64}\left|A \cap I_{u}\right|,
$$

assuming $h \geq 16$. Note that both $\left|A \cap I_{v}\right|$ and $\left|A \cap I_{u}\right|$ may increase. From (5) to (7), $\left|A \cap I_{u}\right|$ must increase by $\Omega\left(\left|A \cap I_{v}\right|\right)=\Omega\left(\left|A \cap I_{u}\right|\right)$; from (5) to (8), $\left|A \cap I_{v}\right|$ must increase by $\Omega\left(\left|A \cap I_{u}\right|\right)$, which implies that $\left|A \cap I_{u}\right|$ must also increase by $\Omega\left(\left|A \cap I_{u}\right|\right)$ since $I_{v} \subset I_{u}$. This means that between two partial rebuildings of $u,\left|A \cap I_{u}\right|$ must have increased by a constant factor. Thus, we can charge the rebuilding cost of $u$ to the $\Omega\left(\left|A \cap I_{u}\right|\right)$ new items that have arrived since the last rebuilding, $O(k /(\epsilon m))$ each. Since each item is contained in the intervals of $O(h)$ nodes, it is charged a cost of $O(h k /(\epsilon m))$ in total. Therefore, the total cost of all the partial rebuildings in this round is $O(h k / \epsilon)=O\left(k / \epsilon \cdot \log \frac{1}{\epsilon}\right)$.

Maintaining the leaves. Finally, we need to make sure that $\left|A \cap I_{v}\right| \leq \frac{\epsilon}{2} m$ for each leaf $v$ as required by the data structure. During initialization, we can easily ensure that $\frac{1}{8} \epsilon m \leq\left|A \cap I_{v}\right| \leq \frac{3}{8} \epsilon m$. During the round, the coordinator monitors $s_{v}$, and will split $v$ by adding two new leaves below $v$ whenever $s_{v}>\left(\frac{\epsilon}{2}-\theta\right) m$. Since $s_{v}$ has error at most $\theta m$, this splitting condition will ensure that $\left|A \cap I_{v}\right| \leq \frac{\epsilon}{2} m$. To split $v$, we again call our initialization algorithm on the interval $I_{v}$, incurring a cost of $O\left(k \frac{\left|A \cap I_{v}\right|}{\epsilon m}\right)=O(k)$. Since we create at most $O(1 / \epsilon)$ leaves in this entire round, the total cost for all the splittings is $O(k / \epsilon)$.

Putting everything together, we obtain the following result.

Theorem 4.1 There is a deterministic algorithm that continuously tracks the $\phi$-quantiles for all $0 \leq \phi \leq 1$ simultaneously and incurs a total communication cost of $O\left(k / \epsilon \cdot \log n \log ^{2} \frac{1}{\epsilon}\right)$.

Implementing with small space. Similar as before, instead of maintaining the counts in the intervals associated with $\mathcal{T}$ exactly at each site, we can again deploy a sketch that maintains the approximate $\epsilon^{\prime}$-quantiles for some $\epsilon^{\prime}=\Theta(\theta)$ to maintain these intervals approximately. Suppose we use the Greenwald-Khanna sketch [18], then we can implement our all-quantile tracking algorithm with $O(1 / \theta \cdot \log (\theta n))=O\left(1 / \epsilon \cdot \log \frac{1}{\epsilon} \log (\epsilon n)\right)$ space per site and amortized $O(\log n)$ time per item.

\section{Open Problems}

We have restricted ourselves to deterministic algorithms in the paper. If randomization is allowed, simple random sampling can be used to achieve a cost of $O\left(\left(k+1 / \epsilon^{2}\right) \cdot \operatorname{polylog}(n, k, 1 / \epsilon)\right)$ for tracking both the heavy hitters and the quantiles. This observation has been well exploited in maintaining the heavy hitters and quantiles for a single stream when both insertions and deletions are present (see e.g. [17]). This breaks the deterministic lower bound for $\epsilon=\omega(1 / k)$. It is not known if randomization can still help for smaller $\epsilon$. Deriving lower bounds for randomized algorithms is also an interesting open problem. Another possible direction is to design algorithms to track the heavy hitters and quantiles within a sliding window in the distributed streaming model. 


\section{References}

[1] P. K. Agarwal and H. Yu. A space-optimal data-stream algorithm for coresets in the plane. In Proc. ACM Symposium on Computational Geometry, 2007.

[2] N. Alon, Y. Matias, and M. Szegedy. The space complexity of approximating the frequency moments. Journal of Computer and System Sciences, 58:137-147, 1999. See also STOC'96.

[3] B. Babcock, S. Babu, M. Datar, R. Motwani, and J. Widom. Models and issues in data stream systems. In Proc. ACM Symposium on Principles of Database Systems, 2002.

[4] B. Babcock and C. Olston. Distributed top-k monitoring. In Proc. ACM SIGMOD International Conference on Management of Data, 2003.

[5] Z. Bar-Yossef, R. Kumar, and D. Sivakumar. Reductions in streaming algorithms, with an application to counting triangles in graphs. In Proc. ACM-SIAM Symposium on Discrete Algorithms, 2002.

[6] G. Cormode and M. Garofalakis. Sketching streams through the net: Distributed approximate query tracking. In Proc. International Conference on Very Large Databases, 2005.

[7] G. Cormode, M. Garofalakis, S. Muthukrishnan, and R. Rastogi. Holistic aggregates in a networked world: Distributed tracking of approximate quantiles. In Proc. ACM SIGMOD International Conference on Management of Data, 2005.

[8] G. Cormode and M. Hadjieleftheriou. Finding frequent items in data streams. In Proc. International Conference on Very Large Databases, 2008.

[9] G. Cormode, F. Korn, S. Muthukrishnan, and D. Srivastava. Space- and time-efficient deterministic algorithms for biased quantiles over data streams. In Proc. ACM Symposium on Principles of Database Systems, 2006.

[10] G. Cormode and S. Muthukrishnan. What's hot and what's not: tracking most frequent items dynamically. In Proc. ACM Symposium on Principles of Database Systems, 2003.

[11] G. Cormode, S. Muthukrishnan, and K. Yi. Algorithms for distributed functional monitoring. In Proc. ACMSIAM Symposium on Discrete Algorithms, 2008.

[12] G. Cormode, S. Muthukrishnan, and W. Zhuang. What's different: Distributed, continuous monitoring of duplicate-resilient aggregates on data streams. In Proc. IEEE International Conference on Data Engineering, pages 20-31, 2006.

[13] G. Cormode, S. Muthukrishnan, and W. Zhuang. Conquering the divide: Continuous clustering of distributed data streams. In Proc. IEEE International Conference on Data Engineering, 2007.

[14] A. Deshpande, C. Guestrin, S. R. Madden, J. M. Hellerstein, and W. Hong. Model-driven data acquisition in sensor networks. In Proc. International Conference on Very Large Databases, 2004.

[15] J. Feigenbaum, S. Kannan, A. McGregor, S. Suri, and J. Zhang. Graph distances in the streaming model: The value of space. In Proc. ACM-SIAM Symposium on Discrete Algorithms, 2005.

[16] R. Fuller and M. Kantardzic. FIDS: Monitoring frequent items over distributed data streams. In MLDM, 2007.

[17] A. C. Gilbert, Y. Kotidis, S. Muthukrishnan, and M. J. Strauss. How to summarize the universe: Dynamic maintenance of quantiles. In Proc. International Conference on Very Large Databases, 2002.

[18] M. Greenwald and S. Khanna. Space-efficient online computation of quantile summaries. In Proc. ACM SIGMOD International Conference on Management of Data, 2001. 
[19] P. Indyk. Algorithms for dynamic geometric problems over data streams. In Proc. ACM Symposium on Theory of Computation, 2004.

[20] P. Indyk and D. Woodruff. Optimal approximations of the frequency moments of data streams. In Proc. ACM Symposium on Theory of Computation, 2005.

[21] A. Jain, J. Hellerstein, S. Ratnasamy, and D. Wetherall. A wakeup call for internet monitoring systems: The case for distributed triggers. In Proceedings of the 3rd Workshop on Hot Topics in Networks (Hotnets), 2004.

[22] R. M. Karp, S. Shenker, and C. H. Papadimitriou. A simple algorithm for finding frequent elements in streams and bags. ACM Transactions on Database Systems, 2003.

[23] R. Keralapura, G. Cormode, and J. Ramamirtham. Communication-efficient distributed monitoring of thresholded counts. In Proc. ACM SIGMOD International Conference on Management of Data, 2006.

[24] A. Manjhi, V. Shkapenyuk, K. Dhamdhere, and C. Olston. Finding (recently) frequent items in distributed data streams. In Proc. IEEE International Conference on Data Engineering, 2005.

[25] G. Manku and R. Motwani. Approximate frequency counts over data streams. In Proc. International Conference on Very Large Databases, 2002.

[26] A. Metwally, D. Agrawal, and A. E. Abbadi. An integrated efficient solution for computing frequent and top-k elements in data streams. ACM Transactions on Database Systems, 2006.

[27] C. Olston, J. Jiang, and J. Widom. Adaptive filters for continuous queries over distributed data streams. In Proc. ACM SIGMOD International Conference on Management of Data, 2003.

[28] C. Olston and J. Widom. Efficient monitoring and querying of distributed, dynamic data via approximate replication. IEEE Data Engineering Bulletin, 2005.

[29] I. Sharfman, A. Schuster, and D. Keren. Shape sensitive geometric monitoring. In Proc. ACM Symposium on Principles of Database Systems, 2008.

[30] S. Suri, C. Toth, and Y. Zhou. Range counting over multidimensional data streams. In Proc. ACM Symposium on Computational Geometry, 2004.

[31] D. Woodruff. Optimal space lower bounds for all frequency moments. In Proc. ACM-SIAM Symposium on Discrete Algorithms, 2004.

[32] A. C. Yao. Some complexity questions related to distributive computing. In Proc. ACM Symposium on Theory of Computation, 1979. 